\title{
Technology Driven Diabetes Management: Application of Cellular Transmission of Glucose Data
}

\author{
John P Bernot*, Manali Patel, Holly Borders, Jade Hairston, Steven Davis and Julienne Kirk \\ Wake Forest Health Sciences, Family \& Community Medicine, Medical Center Boulevard, USA

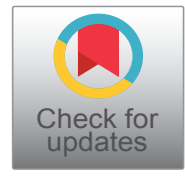

*Corresponding author: John P Bernot, MD, Wake Forest Health Sciences, Family \& Community Medicine, Medical Center Boulevard, USA, Tel: 4105516711,E-mail: jpbernot@gmail.com

\begin{abstract}
Background: Wireless monitoring is an option for patients with diabetes to communicate blood glucose results in realtime. This study evaluates the feasibility of using wireless technology to monitor glucose and to assess the impact on A1C.

Research design and methods: A prospective study was performed in 10 patients with uncontrolled diabetes. Subjects received a wireless glucose monitor with the capability to transmit their glucose measurements to an external server via cellular service. The clinician analyzed these measurements, and communicated with the patient via the glucometer. We examined the impact of increased clinician-patient communication on blood glucose control as measured by SMBG and A1C over a period of 6 months.

Results: After 6 months, a series of 4 questions assessing satisfaction and feasibility resulted in scores between 4.4 and 4.8 out of possible 5 . Nine of the 10 patients had absolute reductions in $\mathrm{A} 1 \mathrm{C}$ at 6 months. Mean $\mathrm{A} 1 \mathrm{C}$ values between baseline and six months demonstrated a statistically significant reduction of $9.8 \%$ versus $8.3 \%(p=0.003)$. No adverse or serious events were reported during the study.

Conclusion: Our study indicates that technology-driven diabetes management using cellular transmission is an easy way to communicate information between patient and provider that can improve $\mathrm{A} 1 \mathrm{C}$.
\end{abstract}

\section{Introduction}

The prevalence of diabetes mellitus in the United States has increased four-fold in the last thirty-six years from approximately 5.5 million to 22 million [1]. WorIdwide, diabetes affects nearly 387 million individuals, and that number is expected to reach half a billion by 2035. Over $\$ 175$ million is spent annually on direct medical costs [2]. Vast resources are dedicated to the monitoring diabetes with many challenges in patient glycemic control.

Clinical inertia has been found to contribute to the potential disconnect between the patient provider to achieve clinical goals. A breakdown in communication between patient and clinician prevents optimal glycemic control and efficient methods for communication are needed to improve patient care [3]. Glucometers are common devices to record and track blood glucose measurements. Patients are encouraged to bring blood glucose logs and meters to appointments to allow assessment of diabetes control by clinicians. Patients frequently fail to bring the requested information to appointments, creating a communication and management barrier. Real-time blood glucose monitoring utilizing wireless technology to communicate health information can help improve communication and data exchange between patient and provider. Wireless technology has demonstrated the potential to improve health outcomes and patient care; however, the widespread use and implementation in clinical practice is still being investigated [4-7].

Telcare ${ }^{\circledR}$ pioneered one of the first cellular-enabled glucometers in 2015, potentially eliminating the patient's responsibility of bringing blood glucose logs to their appointments. The meter uses a cellular signal to transmit data and does not require internet connection $[4,8]$. Thus, it can automatically upload blood glucose readings to a password protected cloud storage server that both the clinician and patient can access. Clinicians can use the readings to provide timely feedback via text 
message alerts embedded into the glucometer. This technological capability to provide real-time data and patient-provider communication has the potential to close the communication gap that significantly contributes to clinical inertia. As such, this pilot study aims to determine the feasibility of using wireless technology in a busy primary care practice to monitor patients' real-time blood-glucose on a weekly basis and its potential impact on glycemic control.

\section{Patients and Methods}

\section{Study design and setting}

This interventional, longitudinal, prospective study was conducted at a primary care practice in the Southeast that employs family medicine physicians, advanced practice providers, and a pharmacist educator who shares responsibility for diabetes care as a certified diabetes educator.

\section{Study population}

Eligible persons were aged 18 years or older and had type 1 or 2 diabetes using a self-monitoring glucose device. Other inclusion criteria included participants with uncontrolled diabetes, as defined by hemoglobin A1c (A1C) between 8 and 11\%. Participants had to regularly attend annual follow-up appointments at the family medicine clinic and demonstrate a history of regularly performed Self-Monitoring of Blood Glucose (SMBG). Subjects were excluded if they did not perform regular SMBG or had a history of more than one "no show" appointments in the 12 months prior to recruitment. A total of 25 candidates were screened by the investigators for inclusion of 10 participants in this pilot study.

\section{Study procedure}

At the initial study visit, each consented participant was provided with a glucose meter and testing supplies. They also receive training materials and a demonstration in the use of the meter. The study meter is capable of transmitting wireless glucose measurements to a protected web site accessible only by user name and password. The investigators are able to see real time glucose values for all participants; however, study participants could only see their individual glucose values. The glucose monitor and supplies were provided free of charge and did not require a live internet connection.

Study participants were asked to check their blood glucose regularly as recommend by the primary care provider (at least daily). Throughout the study, participants were sent triggered messages (i.e. "Your blood sugar is very high. Please call the clinic to discuss".) If glucose levels were $>400$ or $<60 \mathrm{mg} / \mathrm{dL}$, patients were advised to seek immediate assistance. Triggered glucose values were evaluated by study investigators at least weekly. Investigators of the study obtained reports of the SMBG through a secure website that was available only to the clinicians. Two of the study investigators worked with the participants and evaluated the SMBG values. Starting at month one, each time glucose testing was done by a study participant an automated self-management message was triggered. The purpose of these messages was to improve patients' self-management behavior and to encourage patients to actively participate in their diabetes care.

A1C blood tests were obtained at baseline, 3 months, and 6 months. Participants were contacted at the discretion of the clinician on a regular basis to discuss blood glucose control and to adjust diabetes treatment regimen. At the final study visit, clinicians conducted a post-intervention interview to assess patient opinions of the intervention. All procedures were approved by the Wake Forest School of Medicine Institutional Review Board.

\section{Statistical analysis}

Data was analyzed using SPSS (IBM Corp. Released 2016. IBM SPSS Statistics for Windows, Version 24.0. Armonk, NY: IBM Corp). Descriptive statistics were computed for all variables. Satisfaction scores were measured using a five point Likert Scale. Means and standard deviations were calculated for self-monitoring glucose levels, A1C, and satisfaction with meter questions. The SPSS procedure GLM was used in a repeated measures ANOVA to asses A1C levels at baseline and at three months and at six months. Post hoc analysis adjusted for multiple comparisons (Bonferonni).

\section{Results}

\section{Participant characteristics}

A total of 10 participants were recruited for the study. Table 1 includes baseline demographic information. Mean age of the patients was $58.2 \pm 5.4$ years. Women represented two thirds of the participants. Half of the

Table 1: Baseline patient characteristics.

\begin{tabular}{|l|l|}
\hline & Total Sample (n= 10) \\
\hline Age (years) & $58.2 \pm 5.4$ \\
\hline $\begin{array}{l}\text { Gender (\% of total) } \\
\quad \text { Female \% } \\
\quad \text { Male \% }\end{array}$ & 70 \\
\hline Ethnicity & 30 \\
$\quad$ African American \% & 50 \\
$\quad$ Non-Hispanic White \% & 40 \\
$\quad$ Asian or Pacific Islander \% & 10 \\
\hline Baseline Hemoglobin A1C (\%) & $9.8 \pm 1.0$ \\
\hline Baseline BMI & $35.2 \pm-6.1$ \\
\hline Baseline Weight (kg) & $97.4 \pm 18.5$ \\
\hline Diabetes Type (\% of total) & \\
Type 1\% & 20 \\
Type 2\% & 80 \\
\hline
\end{tabular}

Age, BMI, Baseline Hemoglobin A1c, and Baseline weight are expressed as mean \pm SD. Gender, Ethnicity, and Diabetes Type are expressed as percentage of the total. 


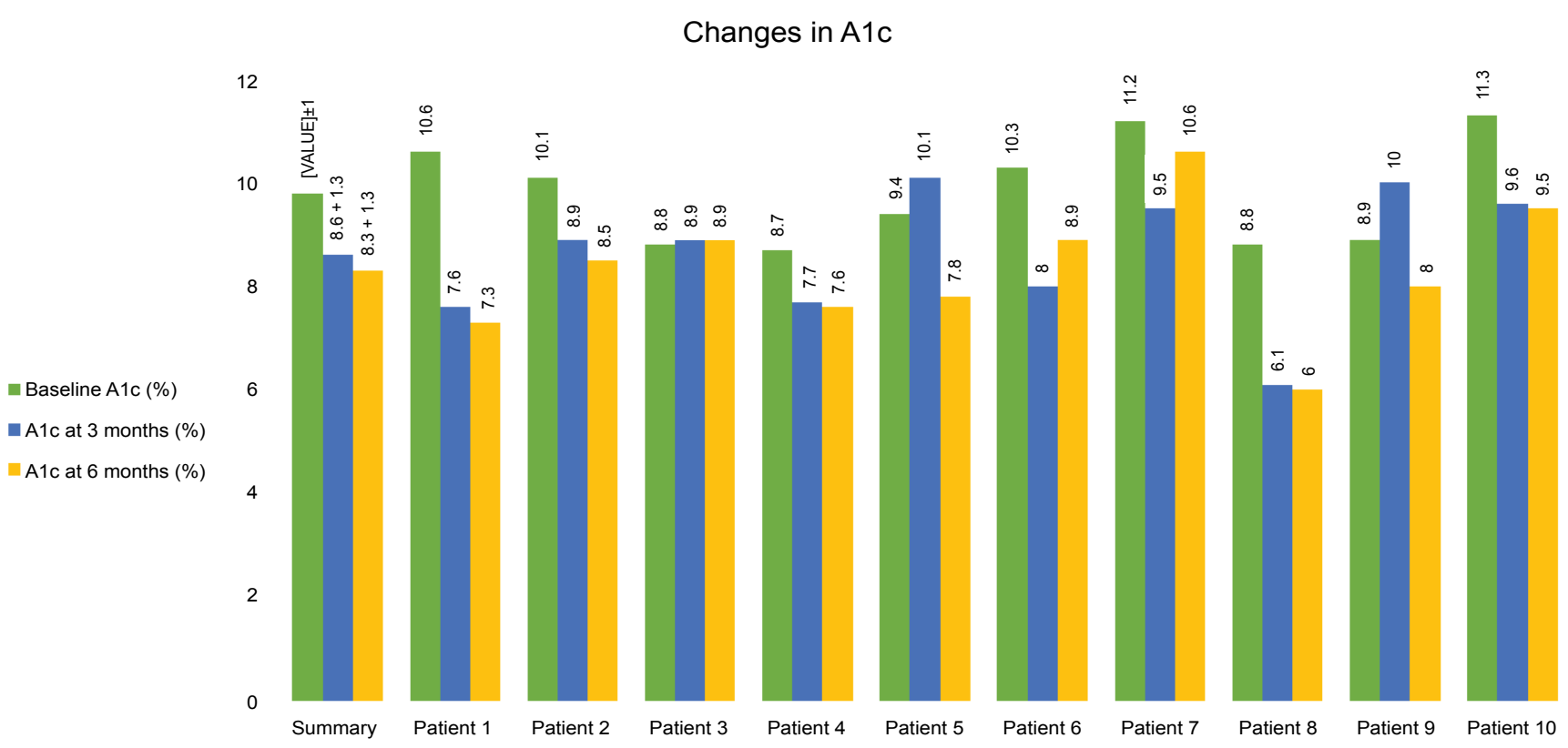

Baseline to 3 months: $p=0.027$; Baseline to 6 months: $p=0.001 ; 3$ to 6 months: $p=0.359$

Figure 1: Changes in $\mathrm{A} 1 \mathrm{C}$.

participants were African American followed by non-Hispanic whites and Asian representation. Baseline A1C was poorly controlled at an average level of $9.8 \pm 1.0 \%$. Seven out of 10 participants were on an insulin-based regimen for glycemic control. Patients with both type 1 and type 2 diabetes were included in the study, with the majority (80\%) being type 2 and obese by an average Body Mass Index (BMI) of $35.2 \pm 6.1$.

\section{Measures}

Participants had an A1C obtained at baseline, three, and six months representing average glucose control over a three-month period. Figure 1 shows poor glycemic control at baseline. At three months, there was a reduction in $\mathrm{A} 1 \mathrm{C}$ by over $1 \%$. The absolute $\mathrm{A} 1 \mathrm{C}$ reduction was found in seven of the ten participants. The mean A1C scores for baseline versus three months $(9.8 \%$ versus $8.6 \%)$ approached significance $(p=0.082)$. There was not a significant difference between levels at three months and six months for A1C (8.6\% versus $8.3 \%$ ) although it was in the predicted direction. There was a significant difference in $\mathrm{A} 1 \mathrm{C}$ between baseline and six months $(9.8 \%$ versus $8.3 \% ; p=0.003)$.

Participants measured daily glucose values at their own set schedule determined by their primary care provider. Average monthly glucose values were captured. Figure 2 results show monthly mean values of SMBG with standard deviations; however, it does not differentiate between fasting versus postprandial values. During the first three months of the study, SMBG testing ranged from 9 to 90 times per month among the ten participants. During the last three months, two patients discontinued using study meters and the range of SMBG testing was two to 77 times per month. Extreme glucose values ( $>400 \mathrm{mg} / \mathrm{dL}$ or $<60 \mathrm{mg} / \mathrm{dL}$ ) were also captured by SMBG and reported during the study; one participant with Type 1 diabetes experiencing an average of 15 episodes per month. Two participants discontinued using the study meter during the study period.

\section{Meter evaluation}

Participants were asked to rate their satisfaction with the study meter on a scale of one to five (one being not satisfied and five being very satisfied). Table 2 contains mean scores for satisfaction between 4.4 and 4.8 with a standard deviation less than one. In addition to the numeric survey responses, participants were asked to respond to a series of questions designed to elicit their experience with the real-time blood glucose monitoring through the use of the meter. All 10 study members participated in the interview. Each participant was asked six open-ended questions as listed in Table 2. The response from participants coalesced around three main themes: (1) The transition to and the ease of use of the new meter; (2) The quality and transmission of blood glucose information; and (3) The experience with the meter's messaging features.

Participants indicated a high satisfaction of the study meter with positive feedback. The first relevant theme that arose from the interview revolved around the transition to and the ease of use of the new meter. "I loved it. I felt very comfortable using it" said one patient. Others stated "Nothing that was any different than using my regular meter" and "I didn't have to keep a glucose log. Before, I would lose my log. It is much easier". In the end, the patients were overall very comfortable with the transition, although two patients described minor difficulties that were quickly resolved. Three patients conveyed negative sentiments regarding the ongoing need to keep the device's battery charged. Several par- 


\section{AVERAGE GLUCOSE FOR SELF-MONITORING BLOOD GLUCOSE READING}

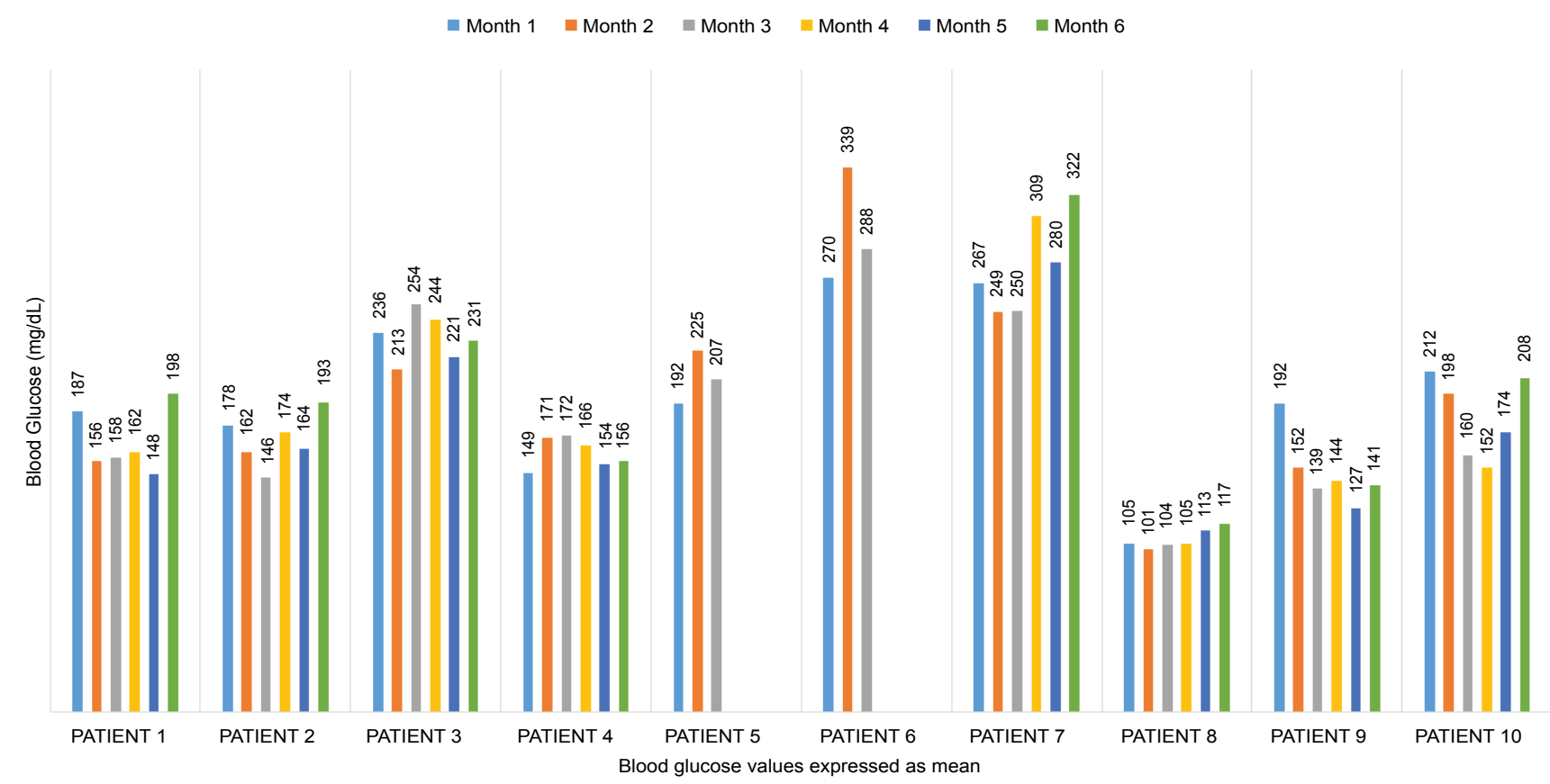

Figure 2: Average glucose for self-monitoring blood glucose readings.

Table 2: Participant meter evaluation.

\section{Survey questions}

1. Have you been satisfied with the meter use?

Total sample $(n=10)$

2. Is the meter easy to use?

$4.4 \pm 0.84$

$4.7 \pm 0.48$

3. How was the transition to this meter from your old meter?

4. How useful did you find the transmission of your blood sugar readings to your healthcare provider? $4.8 \pm 0.42$ Interview questions

1. What is your comfort level with the real-time wireless glucose measurements?

2. How did the real-time glucose measurements affect the quality of the care provided by your clinician?

3. Did utilizing the wireless technology for your glucose reporting require more time for you on a weekly basis compared to your previous meter? If yes, how much?

4. What challenges did you face in using this technology?

5. What benefits did you see in using this technology?

6. What are your thoughts on the feasibility of continuing this intervention long term?

Survey question responses are scored on scale of 1 (not satisfied) to 5 (extremely satisfied) and are expressed as mean \pm SD.

ticipants discussed continuing to use the meter beyond the study with comments such as "I would love to keep the meter!" Despite that, several of those who desired to keep using the meter had concerns about coverage and cost of the test strips.

Participants had a significant amount of comments regarding the quality and transmission of the blood glucose information. The patient experiences are important to assess when using new technology. One participant said "The readings were much higher on this meter than the other meter. I brought it in, but it still wasn't working. I'm now using the old meter". The concern about differences between meters was echoed by two other participants; however, the other seven patients did not have any concerns with the readings. Regarding the transmission of the information, the participants were overwhelmingly positive about the capabilities. One survey response was "The 'real-time' was probably the biggest advantage of using this device. At one time my clinician immediately called me regarding several high blood glucose numbers. We were able to talk through that day's events that led up to the high blood sugar". Another stated "The doctor was able to keep check of my glucose in between doctor visits, which made it easier for her and for me to relate what is happening on a daily basis". In total, eight participants explicitly related a positive experience with the information exchange.

The final theme that emerged was the patient experience with the meter's ability to send educational messages to the participants. Four participants expressed positive feedback including "It tells me directions if too high or too low. I like the meter. Other meters only give 
you a meter". and "I also loved the tips it gave me". No concerns or negative experiences were conveyed regarding the messaging features of the meter.

\section{Discussion}

Real-time glucose monitoring is a dynamic process to efficiently communicate with patients utilizing real-time glucose information. Many SMBG meters have the capability of transmitting data and the impact on health continues to expand to address potential communication barriers between patients and clinicians $[6,9,10]$. The information from this pilot study adds to the body of literature to support the use of real-time SMBG data in a primary care setting to improve clinical outcomes [11-13]. We utilized a meter that can transmit wireless glucose measurements to a protected web site only accessible by user name and password.

The study participants are representative of patients with difficult-to-control diabetes with many insulin-dependent individuals. The investigators are able to see real-time glucose values and actively follow-up on SMBG values. Participants were asked to monitor blood glucose at least daily and many monitored two to three times per day. We found a wide variability among the number of times participants performed SMBG checks. We included Type 1 and 2 patients with diabetes. Two patients discontinued use of the study meter. For one participant, it was due to initiation of an insulin pump that required a different SMBG monitoring system and for the other, it was preference for another meter. The satisfaction survey for the study meter indicates, on average, participants experienced ease of transition from previous monitoring devices to the new meter and had high satisfaction with the additional communication of SMBG data with the healthcare provider.

Another objective of this study was to determine the feasibility of using wireless technology to provide patients' real-time blood glucose data that was easily implemented and accepted by participants. The clinical study investigators were able to respond to the SMBG with time to assess the information before communicating with the participant addressing the issue of clinical inertia and individualizing care [14]. For nine of the ten participants, there was an absolute reduction in A1C. Between three and six months, there was sustainability in the $\mathrm{A} 1 \mathrm{C}$ reductions. At six months, the $\mathrm{A} 1 \mathrm{C}$ change from baseline remained constant and showed statistical significant improvement in glycemic control. The continued improvement in average glucose values for over more than three months is noteworthy.

The providers in the study had actionable data provided through the meter by access to a protected web site. While our pilot study shows feasibility of implementing new technology for management of diabetes, limitations should be noted. The sample size is small but included both men and women with greater than 50\% minority population and an older group of individuals.
We were able to show clinically significant change in A1C at 6 months despite our small sample size and variation in participant testing patterns. The participant sample contained an extreme outlier who experienced wide fluctuations in SMBG that could have negatively impacted our statistical significance. Inclusion of Type 1 and 2 diabetes participants with diabetes may affect the generalizability of the study.

Over a period of six months, we found that at least weekly assessment of participant SMBG and follow-up with electronic communication resulted in improved glycemic outcomes. Specifically, participants received self-management tips that alerted the individual in a message up to 450 characters. A variety of different messages were sent to participants regarding health management and taking care of day to day needs related to diabetes (i.e. SMBG, diet, stress management, and exercise). The automated message may have contributed to the sustained $\mathrm{A} 1 \mathrm{C}$ reduction over six months.

\section{Conclusion}

We found increased patient-provider communication by real time cellular transmission glucose monitoring can clinically improve $\mathrm{A} 1 \mathrm{C}$ with both high patient satisfaction and minimal implementation challenges. This pilot study observed a positive impact of technology driven diabetes management on patients with uncontrolled diabetes. The impact of regular health care messages linked to SMBG warrants further investigation.

\section{References}

1. (2017) Centers for Disease Control and Prevention.

2. (2017) American Diabetes Association.

3. Strain WD, Cos X, Hirst M, Vencio S, Mohan V, et al. (2014) Time to do more: Addressing clinical inertia in the management of type 2 diabetes mellitus. Diabetes Res Clin Pract 105: 302-312.

4. Omre AH (2010) Bluetooth low energy: Wireless connectivity for medical monitoring. J Diabetes Sci Technol 4: 457-463.

5. Dungan K (2014) Monitoring Technologies - Continuous Glucose Monitoring, Mobile Technology, Biomarkers of Glycemic Control. In: De Groot LJ, Chrousos G, Dungan K, Endotext [Internet]. South Dartmouth (MA).

6. Hinnen DA, Buskirk A, Lyden M, Amstutz L, Hunter T, et al. (2015) Use of diabetes data management software reports by health care providers, patients with diabetes, and caregivers improves accuracy and efficiency of data analysis and interpretation compared with traditional logbook data: First results of the Accu-Chek Connect Reports Utility and Efficiency Study (ACCRUES). J Diabetes Sci Technol 9: 293-301.

7. Huang Z, Tao H, Meng Q, Jing L (2015) Management of endocrine disease. Effects of telecare intervention on glycemic control in type 2 diabetes: A systematic review and meta-analysis of randomized controlled trials. Eur J Endocrinol 172: 93-101.

8. Bethesda (2015) Telcare Pioneers Development of the First Cellular-Enabled Glucometer, Secures Patent Allowance for Critical Technology that Supports Improved Diabetes Management. 
9. Nhavoto JA, Grönlund A (2014) Mobile technologies and geographic information systems to improve health care systems: A literature review. JMIR Mhealth Uhealth 2: e21.

10. Klonoff DC (2013) The current status of mHealth for diabetes: Will it be the next big thing? J Diabetes Sci Technol 7: 749-758.

11. Watson AJ, Kvedar JC, Rahman B, Pelletier AC, Salber G, et al. (2009) Diabetes connected health: A pilot study of $a$ patient- and provider-shared glucose monitoring web application. J Diabetes Sci Technol 3: 345-352.
12. Dobson KG, Hall P (2014) A pilot study examining patient attitudes and intentions to adopt assistive technologies into type 2 diabetes self-management. J Diabetes Sci Technol 9: 309-315.

13. Faridi Z, Liberti L, Shuval K, Northrup V, Ali A, et al. (2008) Evaluating the impact of mobile telephone technology on type 2 diabetic patients' self-management: The NICHE pilot study. J Eval Clin Pract 14: 465-469.

14. Strain WD, Bluher M, Paldanius $P$ (2014) Clinical inertia in individualizing care for diabetes: Is there time to do more in type 2 diabetes? Diabetes Ther 5: 347-354. 